\title{
Infantile Hemangiopericytoma: Two Cases Report and Literature Review
}

\author{
Gamal Al-Saied ${ }^{1}$, Amin Abokifa ${ }^{1}$, Mohamed Al-Saeed ${ }^{1}$, Mohamed Abdul Aziz ${ }^{1}$, Medhat Ibrahim ${ }^{1}$, \\ Mohamed Shahin ${ }^{1}$, Tharwat Hussain ${ }^{1}$, Moemen Hafez $^{2}$, Dalal Nemenqani ${ }^{2}$, Naglaa Fawzy ${ }^{3}$ \\ ${ }^{1}$ Departments of Pediatric Surgery, King Abdul Aziz Specialist Hospital, Taif, KSA \\ ${ }^{2}$ Departments of Pathology, King Abdul Aziz Specialist Hospital, Taif, KSA \\ ${ }^{3}$ Departments of Radiology, King Abdul Aziz Specialist Hospital, Taif, KSA \\ E-mail: gamal4a@hotmail.com \\ Received February 14, 2011; revised April 15, 2011; accepted April 23, 2011
}

\begin{abstract}
Hemangiopericytoma is a rare vascular tumour of infants. Although generally considered to be benign, local recurrence and metastases can occur. Herein, we report on two full term girls, delivered with lumbosacral swelling and left thigh swelling respectively. Complete surgical excision with safety margins was performed for each lesion. Histologic examination of both lesions showed picture of infantile hemangiopericytoma. There is no evidence of local recurrence or distant metastasis during last 20 and 17 months for $1^{\text {st }}$ case and $2^{\text {nd }}$ case respectively. In conclusion; most infantile hemangiopericytoma follow a benign course. Rarely these tumours behave aggressively with local infiltration, recurrences and even distant metastases. Careful follow up is therefore essential.
\end{abstract}

Keywords: Infantile Hemangiopericytoma, Vascular Tumour

\section{Introduction}

Hemangiopericytoma is an uncommon tumour of infants which originates from the vascular pericytes, which are contractile cells that spiral around capillary walls. Therefore, hemangiopericytoma have a wide distribution in both soft tissue and skeletal system [1].

Although generally considered to be benign, distant metastases can occur [2]. Histologic examination is the only way to establish the diagnosis of hemangiopericytoma. Hemangiopericytoma is unresponsive to steroid therapy, unlike other vascular malformations, resection is the treatment of choice [2]. The prognosis of the tumour behaviour is still not feasible and it has the potential to demonstrate a highly malignant course [1]. Long-term postoperative follow-up is therefore essential for the early detection of local recurrence and distant metastases [2]. Herein, we report on the outcome of the initial treatment of two full term girls, delivered on January and March 2009 at King Abdul Aziz Specialist Hospitl, Taif, Saudi Arabia; with lumbosacral swelling and left thigh swelling respectively finally diagnosed as infantile hemangiopericytoma by histologic examination.

\section{Case I}

3 day-old, full term girl, $3.5 \mathrm{~kg}$, delivered with lumbosacral swelling. The baby was generally doing fine, pinkish on room air, active and crying. Moving her legs actively. The swelling was measuring $7 \times 6 \mathrm{~cm}$, heterogeneous consistency, firm in some areas and cystic in others, non tender, non reducible, non compressible, non fluctuant. Immovable, fixed to the overlying skin and under lying structures. On auscultation there is no bruit. The skin over it was normal. No lymphadenopathy and no viceromegaly. No other associated congenital anomalies (Figure 1(a)).

CBC and chemistry were within normal limit. Alpha fetoprotein was $(47,000 \mathrm{ng} / \mathrm{ml})$ and b-hCG (3.05). Chest $\mathrm{X}$-rays was normal. CT scan with IV contrast of lumbosacral region showed a well defined oval mass with normal density and sign of calcification of fat or cystic components but there was no sign of bone erosion or defect (Figure 1(b)). MRI of lumbosacral region revealed a well defined oval mass appears normointense on $\mathrm{T}_{1} \mathrm{~W}$ and slight hyperintense on $\mathrm{T}_{2} \mathrm{~W}$ images in the right side of lumbo-sacral canal continued with fibrotic band at sacral region without bone erosions or tethering of the 

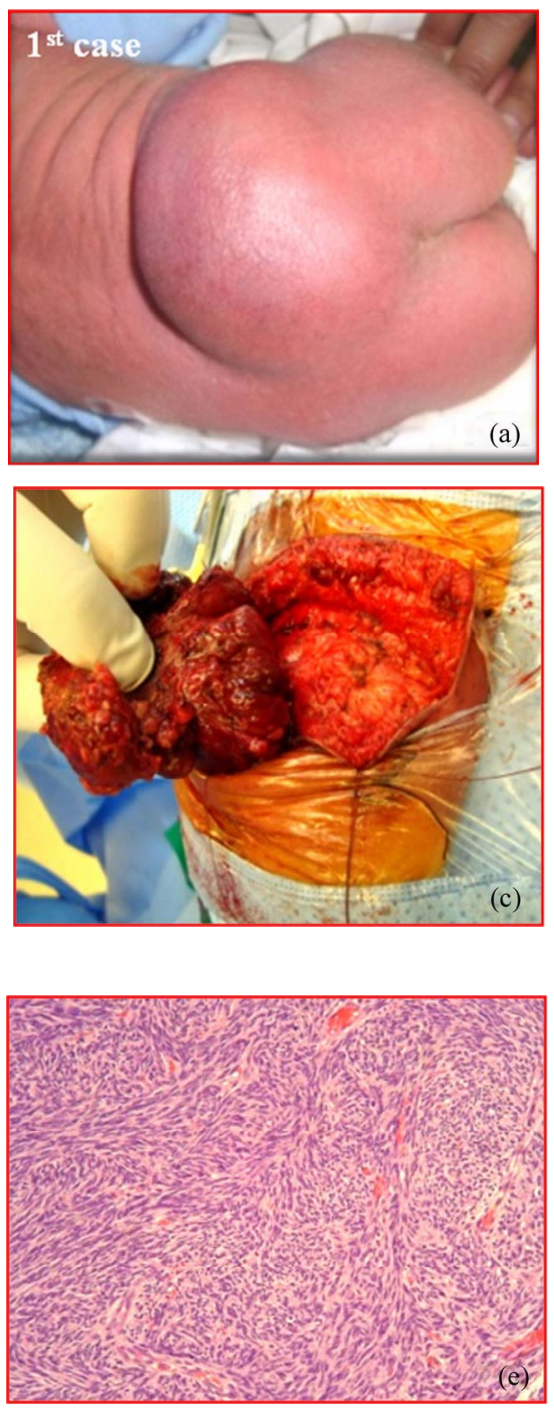
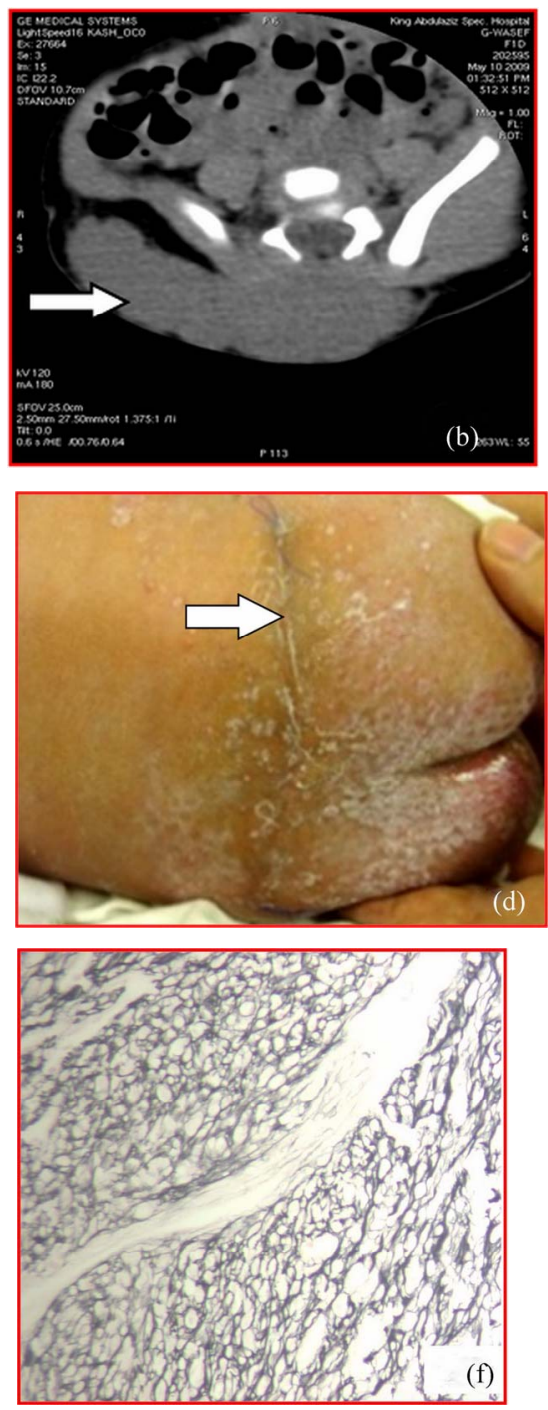

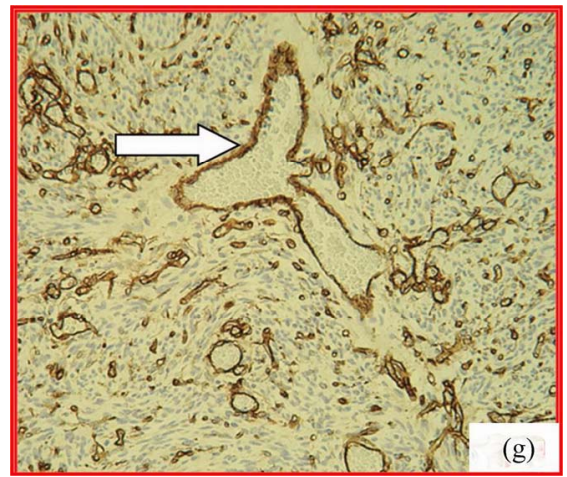

Figure 1. (a) 3 day-old, full term girl, 3.5 kg., delivered with lumbosacral swelling. $7 \times 6 \times 7 \mathrm{~cm}$, heterogeneous consistency, non tender, non reducible, non compressible and non fluctuant; (b) CT with IV contrast of $1^{\text {st }}$ case revealed solid mass in the lumbosacral region without extension to spinal canal (white arrow); (c) Complete surgical excision was done for the lumbosacral mass with safety margins; (d) 1month after surgery, the wound is nicely healed (arrow); (e) Hematoxylin and Eosin stained section showed plump spindle shaped cells with prominent vascular pattern consisting of thin walled branching vessel (antler configuration). (H\&Ex200); (f) Reticulin stained section showed dense reticulin network surround blood vessels and individual tumour cells. (Strept.A.B.tech. $\times$ 400); (g) Immunhistochemistry revealed, Positive $\rightarrow$ Vimentin, Positive $\rightarrow$ CD34 Positive $\rightarrow$ S100, Focal positive $\rightarrow$ Actin (myopericytes), Negative $\rightarrow$ Desmin, Negative $\rightarrow$ Cytokeratin. Branching vascular spaces some of them showed stag horn configuration (arrow). 
cord. Conus medullaris was normal. Initially diagnosed as teratoma versus myelomeningocele.

After induction of general endotracheal anaesthesia, the girl is placed in prone position with the pelvis elevated. The skin of lumbosacral region down to mid-thigh is prepared by cleaning with prewarmed povidone iodine. A single dose of broad spectrum antibiotics is given on induction of anaesthesia. A horizontal skin incision is made over the lumbosacral mass. Then, the upper and lower skin flaps are dissected off the mass using bipolar cautery and optical magnification. The mass was dark red in colour with heterogeneous consistency, firm in some areas and cystic in other areas. Then, the mass is dissected off the fascia lumbodorsalis and the fibrotic band tethering the mass to sacral region. There was no bony defect or connection with dura of lumbosacral canal or tethering of the cord. The subcutaneous tissue is re-approximated with Vicryl 3/0 and the skin closed with subcuticular sutures of Vicryl 4/0 (Figure 1(c) and (d)). The excised mass is sent to histopathological examination. The postoperative course was uneventful. The girl is discharged on the $3^{\text {rd }}$ postoperative day and followed up in outpatient clinic.

The histological and immunhistochemistry features were consisting with infantile hemangiopericytoma (Figure 1(e)-(g)). There was no evidence of local recurrence or distant metastasis during last 20 months.

\section{Case II}

7 day-old full term girl, $4.140 \mathrm{~kg}$, the baby was pinkish on room air, active and crying. Moving her legs actively. Systemic examination was unremarkable. Local examination revealed a firm, violet-coloured swelling on the anterior surface of left thigh, and measuring $5 \times 6 \mathrm{~cm}$. There was marked surface telangiectasia and focal crusting. The surface was easily indented. Non tender to palpation. Non compressible, non fluctuant. Attached to the overlying skin and partially movable over the under lying muscle and fascia. The lesion was non-pulsatile. On auscultation there was no bruit. No lymphadenopathy and no hepatomegaly. No other associated congenital anomalies (Figure 2(a)).

CBC and chemistry were within normal limit. CT angiogram of the pelvis and thigh revealed; a well defined multilocular cystic lesion is seen subcutaneous in the anterior aspect of the left upper thigh. It shows faint enhancement of its wall and septae with no dominant feeding arteries. There are two main draining veins for this lesion, one on its upper medial aspect draining into the left femoral vein, and the other one extends from the lower medial aspect and drains into the left great saphenous vein (Figure 1(b)). Initially diagnosed as a hemangiomatous malformation.

After induction of general endotracheal anaesthesia, the girl is placed in supine position. The skin of left thigh is prepared by cleaning with prewarmed povidone iodine. A single dose of broad spectrum antibiotics is given on induction of anaesthesia. An elliptical transverse skin incision is made around the mass. Then, mass is dissected off the underlying quadriceps femoris muscle using bipolar cautery and optical magnification. On cut section the mass was white-grey in colour and soft in consistency. The upper and lower skin edges are undermined with good hemostasis. The subcutaneous tissue is re-approximated with Vicryl $3 / 0$ and the skin closed with subcuticular sutures of Vicryl 4/0 (Figure 2(c) and (d)). The excised mass is sent to histopathological examination. The girl is discharged on the $3^{\text {rd }}$ postoperative day and followed up in outpatient clinic.

The histological and immunhistochemistry features were consisting with infantile hemangiopericytoma (Figure 2(e)-(g)). There was no evidence of local recurrence or distant metastasis during last 17 months.

Microscopic Description of Both Lesions, Hematoxylin and eosin stained sections revealed plump spindle shaped cells with prominent vascular pattern consisting of thin walled vessels lined by single layer of flattened endothelial cells with gapping vascular spaces, some of them showed stag horn configuration. Increased mitotic activity was noted (Figure 1(e) and Figure 2(e)).

Reticulin stained section showed dense reticulin network surrounding blood vessels and individual tumour cells. Focal myxoid area was noted. Isolated tumour nodules within the fibro pseudocapsule of the main mass were seen (Figure 1(f) and Figure 2(f)).

Immunhistochemistry of Both Lesions revealed, Positive $\rightarrow$ Vimentin, Positive $\rightarrow$ CD34 (endothelial lining of interspersed vascular spaces), Positive $\rightarrow$ S100, Focal positive $\rightarrow$ Actin (myopericytes), Negative $\rightarrow$ Desmin, Negative $\rightarrow$ Cytokeratin. (Figure 1(g) and Figure 2(g)).

The morphological criteria suggested by Enzinger and Smith were used to distinguish a benign from a malignant tumour. The criteria were: large size $(>5 \mathrm{cms})$, increased mitotic rate (4 or more per $10 \mathrm{HPF}$ ), high degree of cellularity, immature and pleomophic tumour cells, foci of hemorrhage and necrosis [3].

These histological and immunhistochemistry features of both lesions were consisting with infantile hemangiopericytoma. In accordance with Enzinger and Smith's criteria both lesions were histologically benign. No evidence of malignancy at the time of examination. 

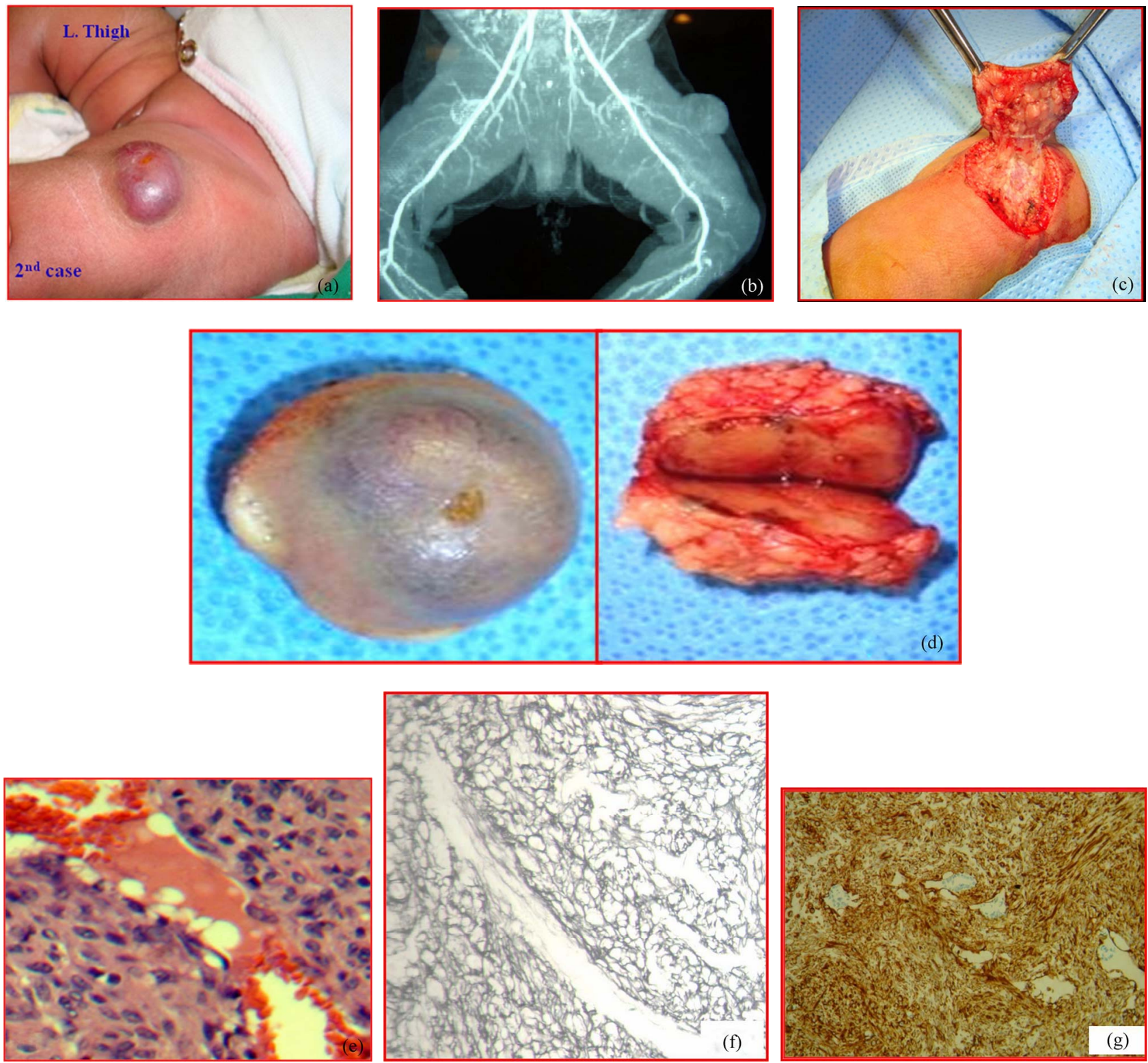

Figure 2. (a) 7 day-old full term girl, delivered with a firm, violet-coloured swelling on the anterior surface of left thigh, measuring $5 \times 6 \mathrm{~cm}$, with marked surface telangiectasia and focal crusting; (b) CT angiogram of pelvis and thigh showing a well defined multilocular cystic lesion. It shows faint enhancement of its wall and septae with no dominant feeding arteries; (c) Complete elliptical surgical excision was performed with safety margins; (d) After complete surgical excision with safety margins, the biopsy was sent for histopathological examination; (e) Hematoxylin and Eosin stained section showed plump spindle shaped cells with prominent vascular pattern consisting of thin walled branching vessel (antler configuration). Increased mitotic activity was noted. (H \& E × 400); (f) Reticulin stained section showed dense reticulin network surround blood vessels and individual tumour cells. (Strept.A.B.tech. $\times$ 400); (g) Immunhistochemistry revealed, Positive $\rightarrow$ Vimentin, Positive $\rightarrow$ CD34 (endothelial lining of interspersed vascular spaces), Positive $\rightarrow$ S100, Focal positive $\rightarrow$ Actin (myopericytes), Negative $\rightarrow$ Desmin, Negative $\rightarrow$ Cytokeratin.

\section{Discussion}

Infantile hemangiopericytoma is a rare vascular tumour in children below one year of age [4]. In 1942, Stout and Murray [5] first described hemangiopericytoma in the lungs as that arising from the vascular pericytes of
Zimmermann which are contractile cells that spiral around capillary walls and differing from a glomus tumour or a hemangioma but they did not differentiate between infantile and adult forms. The infantile type occur in $10 \%$ of cases [6] and in boys more than in girls [7, 8]. Approximately $30 \%$ to $50 \%$ are found in the limbs 
with the remainder either in the head and neck or the trunk. There is seldom discolouration of the lesions in the limb in contrast to those in the head and neck [6]. Infantile hemangiopericytoma tends to be located more superficially than that in adults [9].

In our two cases, both masses were lying subcutaneously, in lumbosacral region without skin discoloration in $1^{\text {st }}$ cases. Whereas, the $2^{\text {nd }}$ mass was violet-coloured on the anterior surface of left thigh.

The diagnosis can be aided by plain radiography which shows a soft-tissue mass, with or without calcific stippling. Ultrasound determines the size of the lesion, vascular loops, the echogenic content and whether or not a capsule is present, and angiography shows the vascular pattern and the presence of feeder vessels. CT and MRI can provide information about the extent of the tumour and help in preoperative planning [9].

MRI and CT scan of lumbo-sacral canal (pre-and post IV contrast) of $1^{\text {st }}$ case incorrectly interpreted the lesion as teratoma versus myelomeningocele.

CT angiogram of pelvis and thigh of $2^{\text {nd }}$ case, initially diagnosed the lesion as a hemangiomatous malformation.

The diagnosis is confirmed by excisional biopsy [9]. The gross appearance of this lesion is usually transparent or white-grey [6]. Feeder vessels may be prominent, and the infantile form tends to be more multilobular than in the adult. Microscopically; the pericyte may be round or oval in shape. The tumour tends to be densely cellular, with prominent vascular channels. Mitotic figures are usually easily identified. The tumour cells stain positively with vimentin [6,9].

In our two cases the final diagnosis was achieved by excisional biopsy. The histological and Immunhistochemistry features were consisting with infantile hemangiopericytoma. In accordance with Enzinger and Smith's criteria [3], both lesions were histologically benign. There was no evidence of malignancy at the time of examination.

The differential diagnosis of congenital hemangiopericytoma includes all tumours which present as a soft tissue mass, including lipoma, hemangioma, and lymphangioma. Some tumours have similar histological features, such as infantile myofibromatosis, synovial sarcoma, fibrosarcoma, malignant fibrous histiocytoma, mesenchymal chondrosarcoma, and leiomyosarcoma [1013]. It is therefore important to examine multiple areas of the specimen to confirm the diagnosis [9].

The management of infantile hemangiopericytoma differs from that in adults, but there is no consensus as to the best method [9]. Wide surgical excision with safety margin is the most accepted method of treatment $[6,14,15]$. That is was our preferred method in management of both cases. Some centres use adjuvant therapy to reduce the risk of local recurrence [6-20] but the value is difficult to assess because of the rarity of these tumours. Chemotherapeutic regimens have included the use of vincristine, doxorubicin, actinomycin and cyclophosphamide. Jha et al. [21] reported success with radiotherapy when there was gross or microscopic evidence of tumour remaining after excision, and adjuvant radiotherapy was used with success by Borg and Benjamin [22]. Other authors have reported that radiotherapy is ineffective $[14,15,23]$. Metastatic disease has only been reported in three cases of congenital hemangiopericytoma $[4,15,24]$ although the adult form of the tumour has a rate of distant metastasis of up to $56 \%$, mainly to the lung and skeleton $[8,25]$. Careful follow up is therefore required. When recurrence does occur, adjunctive chemotherapy has been used successfully [15].

Fortunately, there is no evidence of local recurrence or distant metastasis during last 20 and 17 months in $1^{\text {st }}$ case and $2^{\text {nd }}$ case respectively.

\section{Conclusions}

Most infantile hemangiopericytoma follow a benign course. Rarely these tumours behave aggressively with local infiltration, recurrences and even distant metastases. Careful follow up is therefore essential.

\section{References}

[1] I. Hatzokos, I. Gigis, E. Iosifidou, A. Drakou and J. Pournaras, "Primary Hemangiopericytoma of the Tibia. Treatment by en Bloc Resection and Bone Transportation. A Case Report," Acta orthopaedica Belgica, Vol. 71, 2005, pp. 122-127.

[2] P. V. Bailey, T. R. Weber, T. F. Tracy, D. M. O’Connor and C. Sotelo-Avila, "Congenital Hemangiopericytoma: an Unusual Vascular Neoplasm of Infancy," Bottom of Form Surgery, Vol. 114, 1993, pp. 936-941.

[3] M. J. Hahn, R. Dawnson, J. A. Esterly and D. J. Joseph, "Hemangiopericytoma. An Ultrastructural Study," Cancer, Vol. 31, No1, 1973, pp. 255-261.

[4] S. L. Kauffman and A. P. Stout, "Hemangiopericytoma in Children,” Cancer, Vol. 13, No. 4, 1960, pp. 695-710.

[5] A. P. Stout and M. R. Murray, "Hemangiopericytoma: A Vascular Tumor Featuring Zimmermann's Pericytes," Annals of Surgery, Vol. 116, No. 1, 1942, pp. 26-33. doi:10.1097/00000658-194207000-00004

[6] S. A. Hoey, R. M. Letts and C. Jinemez, "Infantile Hemangiopericytoma of the Musculoskeletal System: Case Report and Literature Review," Journal of Pediatric Orthopaedics, Vol. 18, No. 3, 998, pp. 359-362.

[7] D. L. Baker, D. Oda and R. W. Myall, "Intraoral Infantile Hemangiopericytoma: Literature Review and Addition of a Case," Oral Surgery, Oral Medicine, Oral Pathology, Oral Radiology and Endodontology, Vol. 73, No. 5, 1992, pp. 596-602. 
[8] P. Maillet, A. Lamesch and M. P. Dawagne, "Congenital Hemangiopericytoma,” Chiropractic Pediatrics, Vol. 26, 1985, pp. 22-25.

[9] T. M. O’Donnell, A. T. Devitt, S. Kutty and E. E. Fogarty, "Recurrent Congenital Hemangiopericytoma in a Child," Journal of Bone and Joint Surgery, Vol. 83-B, No. 2, 2001, pp. 269-272.

[10] F. C. Reynolds and W. E. Lansche, "Hemangiopericytoma of the Lower Extremity: A Case Report," Journal of Bone and Joint Surgery, Vol. 40-A, No. 4, 1958, pp. 921-924.

[11] C. M. Coffin and L. P. Dehner, "Fibroblastic-Myofibroblastic Tumours in Children and Adolescents: A Clinico-Pathologic Study of 108 Examples in 103 Patients," Pediatric Pathology, Vol. 11, No. 4, 1991, pp. 569-588. doi:10.3109/15513819109064791

[12] H. Isaacs, "Perinatal (Congenital and Neonatal) Neoplasms: A Report of 110 Cases," Pediatric Pathology, Vol. 3, No. 2-4, 1985, pp. 165-216. doi:10.3109/15513818509078782

[13] T. Mentzel, E. Calonje, A. G. Nascimento and C. D. Fletcher, "Infantile Hemangiopericytoma Versus Infantile Myofibromatosis: Study of a Series Suggesting a Continuous Spectrum of Infantile Myofibroblastic Lesions,” The American Journal of Surgical Pathology, Vol. 18, No. 9, 1994, pp. 922-930. doi:10.1097/00000478-199409000-00007

[14] J. B. Atkinson, G. H. Mahour, H. Isaacs Jr. and J. A. Ortega, "Hemangiopericytoma in Infants and Children: A Report of Six Patients," American Journal of Surgery, Vol. 148, No. 3, 1984, pp. 372-374. doi:10.1016/0002-9610(84)90474-4

[15] P. V. Bailey, T. R. Weber, T. F. Tracy, D. M. O’Connor and C. Sotelo-Avila, "Congenital Hemangiopericytoma: An Unusual Vascular Neoplasm of Infancy,” Surgery, Vol. 114, 1993, pp. 936-941.

[16] J. P. Craven, T. M. Quigley, J. W. Bolen and E. J. Raker, "Current Management and Clinical Outcome of Hemangiopericytomas,” American Journal of Surgery, Vol. 163, No. 5, 1992, pp. 490-493.

\section{doi:10.1016/0002-9610(92)90394-7}

[17] H. Battifora, "Hemangiopericytoma: Ultrastructural Study of Five Cases,” Cancer, Vol. 31, 1973, pp. 1418- 1432.

[18] J. G. Mira, F. C. Chu and J. G. Fortner, "The Role of Radiotherapy in the Management of Malignant Hemangiopericytoma: A Report of Eleven New Cases and a Review of the Literature," Cancer, Vol. 39, 1977, pp. 12541259.

[19] S. Variend, M. Bax and J. van Gorp, “Are Infantile Myofibromatosis, Congenital Fibrosarcoma, and Congenital Hemangiopericytoma Histogenetically Related?” Histopathology, Vol. 26, 1995, pp. 57-62.

[20] P. P. Wong and A. Yagoda, "Chemotherapy of Malignant Hemangiopericytoma,” Cancer, Vol. 41, 1978, pp. 1256-1260.

[21] N. Jha, M. McNeese, H. T. Barkley and J. Kong, "Does Radiotherapy Have a Role in Hemangiopericytoma Management? Report of 14 New Cases and a Review of the Literature," International Journal of Radiation Oncology • Biology • Physics, Vol. 13, No. 9, 1987, pp. 1399-1402.

[22] M. F. Borg and C. S. Benjamin, "A 20 Year Review Of Hemangiopericytoma in Auckland, New Zealand," Clinical oncology (Royal College of Radiologists, Vol. 6, 1994; pp. 371-376.

[23] J. J. Seibert, R. W. Seibert, D. S. Weisenburger and W. Allsbrook, "Multiple Congenital Hemangiopericytomas of the Head and Neck,” Laryngoscope, Vol. 88, 1978, pp. 1006-1012.

[24] A. Morgan and I. Evbuomwan, "Congenital Hemangiopericytoma of the Face with Early Distant Metastasis," Journal of the Royal Colleges of Surgeons of Edinburgh and Ireland, Vol. 28, 1983, pp. 123-125.

[25] E. Salloum, F. Flamant, J. M. Caillaud, et al., "Diagnostic and Therapeutic Problems of Soft Tissue Tumours Other than Rhabdomyosarcoma in Infants under 1 Year of Age: A Clinicopathological Study of 34 Cases Treated at the Institut Gustave-Roussy," Medical and Pediatric Oncology, Vol. 18, 1990, pp. 37-43. doi:10.1002/mpo.2950180108 